\title{
On the generalized Apostol-type Frobenius-Euler polynomials
}

\section{Burak Kurt ${ }^{1}$ and Yilmaz Simsek ${ }^{2 *}$}

${ }^{\text {*Correspondence: }}$

ysimsek63@gmail.com

2Department of Mathematics,

Faculty of Science, Akdeniz

University, Antalya, 07058, Turkey

Full list of author information is

available at the end of the article

\begin{abstract}
The aim of this paper is to derive some new identities related to the Frobenius-Euler polynomials. We also give relation between the generalized Frobenius-Euler polynomials and the generalized Hurwitz-Lerch zeta function at negative integers. Furthermore, our results give generalized Carliz's results which are associated with Frobenius-Euler polynomials.

MSC: $05 \mathrm{~A} 10 ; 11 \mathrm{~B} 65 ; 28 \mathrm{~B} 99 ; 11 \mathrm{~B} 68$

Keywords: Frobenius-Euler polynomials; Hermite-based Frobenius-Euler polynomials; Hermite-based Apostol-Euler polynomials; Apostol-Euler polynomials; Hurwitz-Lerch zeta function
\end{abstract}

\section{Introduction, definitions and notations}

Throughout this presentation, we use the following standard notions: $\mathbb{N}=\{1,2, \ldots\}, \mathbb{N}_{0}=$ $\{0,1,2, \ldots\}=\mathbb{N} \cup\{0\}, \mathbb{Z}^{-}=\{-1,-2, \ldots\}$. Also, as usual $\mathbb{Z}$ denotes the set of integers, $\mathbb{R}$ denotes the set of real numbers and $\mathbb{C}$ denotes the set of complex numbers. Furthermore, $(\lambda)_{0}=1$ and

$$
(\lambda)_{k}=\lambda(\lambda+1)(\lambda+2) \cdots(\lambda+k-1),
$$

where $k \in \mathbb{N}, \lambda \in \mathbb{C}$.

The classical Frobenius-Euler polynomial $H_{n}^{(\alpha)}(x ; u)$ of order $\alpha$ is defined by means of the following generating function:

$$
\left(\frac{1-u}{e^{t}-u}\right)^{\alpha} e^{x t}=\sum_{n=0}^{\infty} H_{n}^{(\alpha)}(x ; u) \frac{t^{n}}{n !}
$$

where $u$ is an algebraic number and $\alpha \in \mathbb{Z}$.

Observe that $H_{n}^{(1)}(x ; u)=H_{n}(x ; u)$, which denotes the Frobenius-Euler polynomials and $H_{n}^{(\alpha)}(0 ; u)=H_{n}^{(\alpha)}(u)$, which denotes the Frobenius-Euler numbers of order $\alpha . H_{n}(x ;-1)=$ $E_{n}(x)$, which denotes the Euler polynomials (cf. [1-24]).

Definition 1.1 (for details, see $[16,17]$ ) Let $a, b, c \in \mathbb{R}^{+}, a \neq b, x \in \mathbb{R}$. The generalized Apostol-type Frobenius-Euler polynomials are defined by means of the following generating function:

$$
\left(\frac{a^{t}-u}{\lambda b^{t}-u}\right)^{\alpha} c^{x t}=\sum_{n=0}^{\infty} \mathcal{H}_{n}^{(\alpha)}(x ; u ; a, b, c ; \lambda) \frac{t^{n}}{n !} .
$$

\section{Springer}

(C) 2013 Kurt and Simsek; licensee Springer. This is an Open Access article distributed under the terms of the Creative Commons Attribution License (http://creativecommons.org/licenses/by/2.0), which permits unrestricted use, distribution, and reproduction in any medium, provided the original work is properly cited. 
Remark 1.2 If we set $x=0$ and $\alpha=1$ in (2), we get

$$
\frac{a^{t}-u}{\lambda b^{t}-u}=\sum_{n=0}^{\infty} \mathcal{H}_{n}(u ; a, b, c ; \lambda) \frac{t^{n}}{n !}
$$

where $\mathcal{H}_{n}(u ; \lambda ; a, b, c)$ denotes the generalized Apostol-type Frobenius-Euler numbers ( $c f$. [17]).

\section{New identities}

In this section, we derive many new identities related to the generalized Apostol-type Frobenius-Euler numbers and polynomials of order $\alpha$.

Theorem 2.1 Let $\alpha, \beta \in \mathbb{Z}$. Each of the following relationships holds true:

$$
\begin{aligned}
& \mathcal{H}_{n}^{(\alpha)}(x ; u ; a, b, c ; \lambda)=\sum_{k=0}^{n}\left(\begin{array}{l}
n \\
k
\end{array}\right) \mathcal{H}_{k}^{(\alpha)}(u ; a, b, c ; \lambda)(x \ln c)^{n-k}, \\
& \mathcal{H}_{n}^{(\alpha+\beta)}(x+y ; u ; a, b, c ; \lambda)=\sum_{k=0}^{n}\left(\begin{array}{l}
n \\
k
\end{array}\right) \mathcal{H}_{k}^{(\alpha)}(x ; u ; a, b, c ; \lambda) \mathcal{H}_{n-k}^{(\beta)}(y ; u ; a, b, c ; \lambda), \\
& ((x+y) \ln c)^{n}=\sum_{k=0}^{n}\left(\begin{array}{l}
n \\
k
\end{array}\right) \mathcal{H}_{n-k}^{(\alpha)}(y ; u ; a, b, c ; \lambda) \mathcal{H}_{k}^{(-\alpha)}(x ; u ; a, b, c ; \lambda),
\end{aligned}
$$

and

$$
\mathcal{H}_{n}^{(-\alpha)}\left(x ; u^{2} ; a^{2}, b^{2}, c^{2} ; \lambda^{2}\right)=\sum_{k=0}^{n}\left(\begin{array}{l}
n \\
k
\end{array}\right) \mathcal{H}_{k}^{(-\alpha)}(x ; u ; a, b, c ; \lambda) \mathcal{H}_{n-k}^{(-\alpha)}(x ;-u ; a, b, c ; \lambda)
$$

Proof of (6) From (2),

$$
\sum_{n=0}^{\infty} \mathcal{H}_{n}^{(-\alpha)}(x ; u ; a, b, c ; \lambda) \frac{t^{n}}{n !} \sum_{n=0}^{\infty} \mathcal{H}_{n}^{(\alpha)}(y ; u ; a, b, c ; \lambda) \frac{t^{n}}{n !}=c^{(x+y) t}
$$

Therefore,

$$
\sum_{n=0}^{\infty}\left(\sum_{k=0}^{n}\left(\begin{array}{l}
n \\
k
\end{array}\right) \mathcal{H}_{n-k}^{(\alpha)}(y ; u ; a, b, c ; \lambda) \mathcal{H}_{k}^{(-\alpha)}(x ; u ; a, b, c ; \lambda)\right) \frac{t^{n}}{n !}=\sum_{n=0}^{\infty}(x \ln c)^{n} \frac{t^{n}}{n !}
$$

Thus, by using the Cauchy product in (8) and then equating the coefficients of $\frac{t^{n}}{n !}$ on both sides of the resulting equation, we obtain the desired result.

The proofs of (4), (5) and (7) are the same as that of (2), thus we omit them.

Observe that in (6) we have

$$
((x+y) \ln c)^{n}=\left(\mathcal{H}^{(\alpha)}(y ; u ; a, b, c ; \lambda)+\mathcal{H}^{(-\alpha)}(x ; u ; a, b, c ; \lambda)\right)^{n}
$$

where $\left(\mathcal{H}^{(\alpha)}(y ; u ; a, b, c ; \lambda)\right)^{n}$ is replaced by $\mathcal{H}_{n}^{(\alpha)}(y ; u ; a, b, c ; \lambda)$. 
Theorem 2.2 Let $\alpha \in \mathbb{N}$. Then we have

$$
\sum_{k=0}^{\alpha}\left(\begin{array}{l}
\alpha \\
k
\end{array}\right)(-u)^{\alpha-k}(x \ln c+k \ln a)^{n}=\sum_{p=0}^{n} \sum_{k=0}^{\alpha}\left(\begin{array}{l}
n \\
p
\end{array}\right)\left(\begin{array}{l}
\alpha \\
k
\end{array}\right)(-u)^{\alpha-k}(k \ln b)^{p} \mathcal{H}_{n-p}^{(\alpha)}(x ; u ; a, b, c ; \lambda) .
$$

Proof By using (2), we get

$$
\begin{aligned}
& \sum_{n=0}^{\infty}\left(\sum_{k=0}^{\alpha}\left(\begin{array}{l}
\alpha \\
k
\end{array}\right)(-u)^{\alpha-k}(x \ln c+k \ln a)^{n}\right) \frac{t^{n}}{n !} \\
& \quad=\sum_{n=0}^{\infty}\left(\sum_{p=0}^{n} \sum_{k=0}^{\alpha}\left(\begin{array}{l}
n \\
p
\end{array}\right)\left(\begin{array}{l}
\alpha \\
k
\end{array}\right)(-u)^{\alpha-k}(k \ln b)^{p} \mathcal{H}_{n-p}^{(\alpha)}(x ; u ; a, b, c ; \lambda)\right) \frac{t^{n}}{n !} .
\end{aligned}
$$

By equating the coefficients of $\frac{t^{n}}{n !}$ on both sides of the resulting equation, we obtain the desired result.

Theorem 2.3 The following relationship holds true:

$$
\begin{aligned}
(2 u-1) & \sum_{r=0}^{n}\left(\begin{array}{l}
n \\
r
\end{array}\right) \mathcal{H}_{r}(x ; u ; a, b, c ; \lambda) \mathcal{H}_{n-r}(y ; 1-u ; a, b, c ; \lambda) \\
= & (u-1) \mathcal{H}_{n}(x+y ; u ; a, b, c ; \lambda)+u \mathcal{H}_{n}(x+y ; 1-u ; a, b, c ; \lambda) \\
& +\sum_{k=0}^{n}\left(\begin{array}{l}
n \\
k
\end{array}\right) \mathcal{H}_{k}(x+y ; u ; a, b, c ; \lambda) \\
& -\sum_{k=0}^{n}\left(\begin{array}{l}
n \\
k
\end{array}\right)(\ln a)_{k}^{n-k} \mathcal{H}(x+y ; 1-u ; a, b, c ; \lambda) .
\end{aligned}
$$

Proof We set

$$
\begin{aligned}
& (2 u-1) \frac{a^{t}-u}{\lambda b^{t}-u} c^{x t} \frac{a^{t}-(1-u)}{\lambda b^{t}-(1-u)} c^{y t} \\
& \quad=\left(a^{t}-u\right)\left(a^{t}-(1-u)\right) c^{(x+y) t}\left(\frac{1}{\lambda b^{t}-u}-\frac{1}{\lambda b^{t}-(1-u)}\right) .
\end{aligned}
$$

From the above equation, we see that

$$
\begin{aligned}
& (2 u-1)\left(\sum_{n=0}^{\infty} \mathcal{H}_{n}(x ; u ; a, b, c ; \lambda) \frac{t^{n}}{n !}\right)\left(\sum_{n=0}^{\infty} \mathcal{H}_{n}(y ; 1-u ; a, b, c ; \lambda) \frac{t^{n}}{n !}\right) \\
& =\left(a^{t}-1+u\right) \sum_{n=0}^{\infty} \mathcal{H}_{n}(x+y ; u ; a, b, c ; \lambda) \frac{t^{n}}{n !}-\left(a^{t}-u\right) \sum_{n=0}^{\infty} \mathcal{H}_{n}(x+y ; 1-u ; a, b, c ; \lambda) \frac{t^{n}}{n !} .
\end{aligned}
$$

Therefore,

$$
\begin{aligned}
& (2 u-1) \sum_{n=0}^{\infty} \sum_{r=0}^{n}\left(\begin{array}{l}
n \\
r
\end{array}\right) \mathcal{H}_{r}(x ; u ; a, b, c ; \lambda) \mathcal{H}_{n-r}(y ; 1-u ; a, b, c ; \lambda) \frac{t^{n}}{n !} \\
& =(u-1) \sum_{n=0}^{\infty} \mathcal{H}_{n}(x+y ; u ; a, b, c ; \lambda) \frac{t^{n}}{n !}+u \sum_{n=0}^{\infty} \mathcal{H}_{n}(x+y ; 1-u ; a, b, c ; \lambda) \frac{t^{n}}{n !}
\end{aligned}
$$




$$
\begin{aligned}
& +\sum_{n=0}^{\infty} \sum_{r=0}^{n}\left(\begin{array}{l}
n \\
r
\end{array}\right)(\ln a)^{n-r} \mathcal{H}_{r}(x+y ; u ; a, b, c ; \lambda) \frac{t^{n}}{n !} \\
& -\sum_{n=0}^{\infty} \sum_{r=0}^{n}\left(\begin{array}{l}
n \\
r
\end{array}\right)(\ln a)^{n-r} \mathcal{H}_{r}(x+y ; 1-u ; a, b, c ; \lambda) \frac{t^{n}}{n !}
\end{aligned}
$$

Comparing the coefficients of $\frac{t^{n}}{n !}$ on both sides of the above equation, we arrive at the desired result.

Remark 2.4 By substituting $a=1, b=c=e, \lambda=1$ into Theorem 2.3, we get Carlitz's results (for details, see [1, Eq. 2.19]) as follows:

$$
\begin{aligned}
& (2 u-1) \sum_{r=0}^{n}\left(\begin{array}{l}
n \\
r
\end{array}\right) H_{r}(x ; u) H_{n-r}(y ; 1-u) \\
& =(u-1) H_{n}(x+y ; u)+u H_{n}(x+y ; 1-u)+H_{n}(x+y ; u)-H_{n}(x+y ; 1-u) .
\end{aligned}
$$

We give the following generating function of the polynomials $Y_{n}(x ; \lambda ; a)$ :

$$
\frac{t}{\lambda a^{t}-1} a^{x t}=\sum_{n=0}^{\infty} Y_{n}(x ; \lambda ; a) \frac{t^{n}}{n !} \quad(a \geq 1)
$$

$(c f .[16,17])$. We also note that

$$
Y_{n}(0 ; \lambda ; a)=Y_{n}(\lambda ; a)
$$

If we substitute $x=0$ and $a=1$ into (10), we see that

$$
Y_{n}(\lambda ; 1)=\frac{1}{\lambda-1}
$$

Theorem 2.5 The generalized Apostol-type Frobenius-Euler polynomial holds true as follows:

$$
\begin{aligned}
n\left(\mathcal{H}_{n}(x ; u ; a, b, b ; \lambda)-\ln \left(c^{x}\right) \mathcal{H}_{n}(x ; u ; a, b, c ; \lambda)\right) \\
=\ln a^{\frac{1}{u}} \sum_{k=0}^{n}\left(\begin{array}{l}
n \\
k
\end{array}\right) Y_{n-k}\left(1 ; \frac{1}{u} ; a\right) \mathcal{H}_{k}(x ; u ; a, b, b ; \lambda) \\
\quad+\ln b^{\frac{\lambda}{u}} \sum_{k=0}^{n}\left(\begin{array}{l}
n \\
k
\end{array}\right) Y_{n-k}\left(\frac{1}{u} ; a\right) \mathcal{H}_{k}^{(2)}(x ; u ; a, b, b ; \lambda) .
\end{aligned}
$$

Proof Substituting $c=b$ for $\alpha=1$ into (2) and taking derivative with respect to $t$, we obtain

$$
\begin{aligned}
& \sum_{n=0}^{\infty} \mathcal{H}_{n+1}(x ; u ; a, b, b ; \lambda) \frac{t^{n}}{n !} \\
& \quad=\frac{a^{t} \ln a}{a^{t}-u} \frac{a^{t}-u}{\lambda b^{t}-u} b^{x t}+\frac{\ln b \lambda b^{t}}{a^{t}-u}\left(\frac{a^{t}-u}{\lambda b^{t}-u}\right)^{2} b^{x t}+\ln \left(b^{x}\right) \frac{a^{t}-u}{\lambda b^{t}-u} b^{x t}
\end{aligned}
$$


Using (10), we have

$$
\begin{aligned}
\sum_{n=0}^{\infty} \mathcal{H}_{n+1}(x ; u ; a, b, b ; \lambda) \frac{t^{n}}{n !}= & \frac{\ln \left(a^{\frac{1}{u}}\right)}{t} \sum_{n=0}^{\infty} \sum_{k=0}^{n}\left(\begin{array}{l}
n \\
k
\end{array}\right) Y_{n-k}\left(1 ; \frac{1}{u} ; a\right) \mathcal{H}_{k}(x ; u ; a, b, b ; \lambda) \frac{t^{n}}{n !} \\
& +\frac{\ln \left(b^{\frac{\lambda}{u}}\right)}{t} \sum_{n=0}^{\infty} \sum_{k=0}^{n}\left(\begin{array}{l}
n \\
k
\end{array}\right) Y_{n-k}\left(\frac{1}{u} ; a\right) \mathcal{H}_{k}^{(2)}(x ; u ; a, b, b ; \lambda) \frac{t^{n}}{n !} \\
& +\ln \left(b^{x}\right) \sum_{n=0}^{\infty} \mathcal{H}_{n}(x ; u ; a, b, b ; \lambda) \frac{t^{n}}{n !} .
\end{aligned}
$$

Thus, after some elementary calculations, we arrive at (11).

Theorem 2.6 Let $|u|<1$ and $m \in \mathbb{N}$. Then we have

$$
\mathcal{H}^{(-m)}(u ; a, b, c ; \lambda)=\sum_{k=0}^{n}\left(\begin{array}{l}
n \\
k
\end{array}\right) \mathcal{H}_{k}^{(-\alpha)}(-x ; u ; a, b, c ; \lambda) \mathcal{H}_{n-k}^{(\alpha-m)}(x ; u ; a, b, c ; \lambda) .
$$

Proof In (2), we replace $\alpha$ by $-\alpha$, then we set

$$
\left(\frac{a^{t}-u}{\lambda b^{t}-u}\right)^{-\alpha} c^{(-x) t} \sum_{n=0}^{\infty} \mathcal{H}_{n}^{(\alpha-m)}(x ; u ; a, b, c ; \lambda) \frac{t^{n}}{n !}=\left(\frac{a^{t}-u}{\lambda b^{t}-u}\right)^{-m} .
$$

By using (2), we get

$$
\sum_{n=0}^{\infty} \mathcal{H}_{n}^{(-\alpha)}(-x ; u ; a, b, c ; \lambda) \frac{t^{n}}{n !} \sum_{n=0}^{\infty} \mathcal{H}_{n}^{(\alpha-m)}(x ; u ; a, b, c ; \lambda) \frac{t^{n}}{n !}=\sum_{n=0}^{\infty} \mathcal{H}_{n}^{(-m)}(u ; a, b, c ; \lambda) \frac{t^{n}}{n !}
$$

Therefore,

$$
\sum_{n=0}^{\infty} \sum_{k=0}^{n}\left(\begin{array}{l}
n \\
k
\end{array}\right) \mathcal{H}_{k}^{(-\alpha)}(-x ; u ; a, b, c ; \lambda) \mathcal{H}_{n-k}^{(\alpha-m)}(x ; u ; a, b, c ; \lambda) \frac{t^{n}}{n !}=\sum_{n=0}^{\infty} \mathcal{H}_{n}^{(-m)}(u ; a, b, c ; \lambda) \frac{t^{n}}{n !}
$$

Comparing the coefficients of $\frac{t^{n}}{n !}$ on both sides of the above equation, we arrive at (12).

\section{Interpolation function}

In this section, we give a recurrence relation between the generalized Frobenius-Euler polynomials and the Hurwitz-Lerch zeta function. Recently, many authors have studied not only the Hurwitz-Lerch zeta function, but also its generalizations, for example (among others), Srivastava [19], Srivastava and Choi [24] and also Garg et al. [6]. The generalization of the Hurwitz-Lerch zeta function $\Phi(z, s, a)$ is given as follows:

$$
\Phi_{\mu, \nu}^{(\rho, \sigma)}(z, s, a):=\sum_{n=0}^{\infty} \frac{(\mu)_{\rho n}}{(\nu)_{\sigma n}} \frac{z^{n}}{(n+a)^{s}}
$$


$\left(\mu \in \mathbb{C}, a, v \in \mathbb{C} \backslash \mathbb{Z}_{0}^{-}, \rho, \sigma \in \mathbb{R}^{+}, \rho<\sigma\right.$ when $s, z \in \mathbb{C}(|z|<1) ; \rho=\sigma$ and $\Re(s-\mu+\nu)>0$ when $|z|=1)$. It is obvious that

$$
\Phi_{\mu, 1}^{(1,1)}(z, s, a)=\Phi_{\mu}^{*}(z, s, a)=\sum_{n=0}^{\infty} \frac{(\mu)_{n}}{n !} \frac{z^{n}}{(n+a)^{s}}
$$

and

$$
\Phi_{n}^{*}(z, s, a)=\sum_{n=0}^{\infty} \frac{(n)_{n}}{n !} \frac{z^{n}}{(n+a)^{s}}=\Phi(z, s, a)
$$

where $\Phi(z, s, a)$ denotes the Lerch-Zeta function ( $c f .[6,19,21,24])$.

Relation between the generalized Apostol-type Frobenius-Euler polynomials and the Hurwitz-Lerch zeta function is given as follows.

Theorem 3.1 Let $\left|\frac{\lambda}{u}\right|<1$. We have

$$
\mathcal{H}_{n}^{(\alpha)}(x ; u ; a, b, c ; \lambda)=\sum_{k=0}^{\alpha}\left(\begin{array}{l}
\alpha \\
k
\end{array}\right)(-u)^{\alpha-k-1} \mathfrak{G}\left(-n ; x, \frac{\lambda}{u} ; a, b, c ; \alpha, k\right)
$$

where

$$
\mathfrak{G}(s ; x, \beta ; a, b, c ; \alpha, j)=\sum_{m=0}^{\infty}\left(\begin{array}{c}
m+\alpha-1 \\
m
\end{array}\right) \frac{\beta^{m}}{(x \ln c+j \ln a+m \ln b)^{s}}, \quad|\beta|<1 .
$$

Proof From (2), we have

$$
\sum_{n=0}^{\infty} \mathcal{H}_{n}^{(\alpha)}(x ; u ; a, b, c ; \lambda) \frac{t^{n}}{n !}=\sum_{j=0}^{\alpha}\left(\begin{array}{c}
\alpha \\
j
\end{array}\right)(-u)^{\alpha-j-1} \sum_{m=0}^{\infty}\left(\begin{array}{c}
m+\alpha-1 \\
m
\end{array}\right)\left(\frac{\lambda}{u}\right)^{m} e^{\alpha(x \ln c+k \ln a+m \ln b)} .
$$

Therefore,

$$
\begin{aligned}
& \sum_{n=0}^{\infty} \mathcal{H}_{n}^{(\alpha)}(x ; u ; a, b, c ; \lambda) \frac{t^{n}}{n !} \\
& \quad=\sum_{n=0}^{\infty} \sum_{k=0}^{\alpha}\left(\begin{array}{l}
\alpha \\
k
\end{array}\right)(-u)^{\alpha-k-1} \sum_{m=0}^{\infty}\left(\begin{array}{c}
m+\alpha-1 \\
m
\end{array}\right)\left(\frac{\lambda}{u}\right)^{m}(x \ln c+k \ln a+m \ln b)^{n} \frac{t^{n}}{n !} .
\end{aligned}
$$

Comparing the coefficients of $\frac{t^{n}}{n !}$ on both sides of the above equation, we have arrive at (14).

Remark 3.2 By substituting $a=1, b=c=e$ into (14), we have

$$
\mathcal{H}_{n}^{(\alpha)}(x ; u ; \lambda)=-\frac{(1-u)^{\alpha}}{u} \mathfrak{G}\left(-n ; x, \frac{\lambda}{u} ; 1, e, e ; \alpha, 1\right)=-\frac{(1-u)^{\alpha}}{u} \Phi\left(\frac{\lambda}{u},-n, x\right),
$$

where

$$
\mathfrak{G}\left(-n ; x, \frac{\lambda}{u} ; 1, e, e ; \alpha, 1\right)=\Phi\left(\frac{\lambda}{u},-n, x\right) .
$$


Remark 3.3 The function $\mathfrak{G}(s ; x, \beta ; a, b, c ; \alpha, j)$ is an interpolation function of the generalized Apostol-type Frobenius-Euler polynomials of order $\alpha$ at negative integers, which is given by the analytic continuation of the $\mathfrak{G}(s ; x, \beta ; a, b, c ; \alpha, j)$ for $s=-n, n \in \mathbb{N}$.

\section{Relations between Array-type polynomials, Apostol-Bernoulli polynomials and generalized Apostol-type Frobenius-Euler polynomial}

In [17], Simsek constructed the generalized $\lambda$-Stirling type numbers of the second kind $\mathcal{S}(n, v ; a, b ; \lambda)$ by means of the following generating function:

$$
f_{S, v}(t ; a, b ; \lambda)=\frac{\left(\lambda b^{t}-a^{t}\right)^{v}}{v !}=\sum_{n=0}^{\infty} \mathcal{S}(n, v ; a, b ; \lambda) \frac{t^{n}}{n !} .
$$

The generating function for these polynomials $\mathcal{S}_{v}^{n}(x ; a, b ; \lambda)$ is given by

$$
g_{v}(x, t ; a, b ; \lambda)=\frac{1}{v !}\left(\lambda b^{t}-a^{t}\right)^{v} b^{x t}=\sum_{n=0}^{\infty} \mathcal{S}_{v}^{n}(x ; a, b ; \lambda) \frac{t^{n}}{n !}
$$

(cf. [17]).

The generalized Apostol-Bernoulli polynomials were defined by Srivastava et al. [22, p.254, Eq. (20)] as follows.

Let $a, b, c \in \mathbb{R}^{+}$with $a \neq b, x \in \mathbb{R}$ and $n \in \mathbb{N}_{0}$. Then the generalized Bernoulli polynomials $\mathfrak{B}_{n}^{(\alpha)}(x ; \lambda ; a, b, c)$ of order $\alpha \in \mathbb{Z}$ are defined by means of the following generating functions:

$$
f_{B}(x, a, b, c ; \lambda ; \alpha)=\left(\frac{t}{\lambda b^{t}-a^{t}}\right)^{\alpha} c^{x t}=\sum_{n=0}^{\infty} \mathfrak{B}_{n}^{(\alpha)}(x ; \lambda ; a, b, c) \frac{t^{n}}{n !}
$$

where

$$
\left|t \ln \left(\frac{a}{b}\right)+\ln \lambda\right|<2 \pi
$$

We note that $\mathfrak{B}_{n}^{(1)}(x ; \lambda ; a, b, c)=\mathfrak{B}_{n}(x ; \lambda ; a, b, c)$ and also $\mathfrak{B}_{n}(x ; \lambda ; 1, e, e)=B_{n}(x ; \lambda)$, which denotes the Apostol-Bernoulli polynomials (cf. [1-24]).

Theorem 4.1 Let $v$ be an integer. Then we have

$$
\mathcal{H}_{n-v}^{(-v)}(x ; u ; a, b, c ; \lambda)=\frac{v !}{u^{2 v}(n)_{v}} \sum_{k=0}^{n}\left(\begin{array}{l}
n \\
k
\end{array}\right) \mathcal{S}_{v}^{n}\left(x, 1, b ; \frac{\lambda}{u}\right) Y_{n-k}^{(v)}\left(\frac{1}{u} ; a\right)
$$

Proof Replacing $c$ by $b$ in (2) and after some calculations, we have

$$
\sum_{n=0}^{\infty} \mathcal{H}_{n}^{(-v)}(x ; u ; a, b, b ; \lambda) \frac{t^{n+v}}{n !}=\frac{v !}{u^{2 v}} \sum_{n=0}^{\infty} S_{v}^{n}\left(x, 1, b ; \frac{\lambda}{u}\right) \frac{t^{n}}{n !} \sum_{n=0}^{\infty} Y_{n}^{(v)}\left(\frac{1}{u} ; a\right) \frac{t^{n}}{n !}
$$

Comparing the coefficients of $\frac{t^{n}}{n !}$ on both sides of the above equation, we arrive at the desired result. 


\section{Corollary 4.2}

$$
\mathcal{H}_{n-v}^{(-v)}(x ; u ; a, b, c ; \lambda)=\frac{v !}{u^{2 v}(n)_{\alpha}} \sum_{k=0}^{n}\left(\begin{array}{l}
n \\
k
\end{array}\right) \mathcal{S}\left(k, v, 1, b ; \frac{\lambda}{u}\right) \mathfrak{B}_{n-k}\left(x, a, b ; \frac{\lambda}{u}\right) .
$$

Proof Replacing $c$ by $b$ in (2) and after some calculations, we have

$$
\sum_{n=0}^{\infty} \mathcal{H}_{n-v}^{(-v)}(x ; u ; a, b, b ; \lambda) \frac{t^{n+v}}{n !}=\frac{v !}{u^{2 v}} \sum_{n=0}^{\infty} \mathcal{S}\left(n, v, 1, b ; \frac{\lambda}{u}\right) \frac{t^{n}}{n !} \sum_{n=0}^{\infty} \mathfrak{B}_{n}\left(x, a, b ; \frac{\lambda}{u}\right) \frac{t^{n}}{n !}
$$

Comparing the coefficients of $\frac{t^{n}}{n !}$ on both sides of the above equation, we arrive at the desired result.

\section{Competing interests}

The authors declare that they have no competing interests.

Authors' contributions

All authors completed the paper together. All authors read and approved the final manuscript.

\section{Author details}

${ }^{1}$ Department of Mathematics, Faculty of Education, Akdeniz University, Antalya, 07058, Turkey. ${ }^{2}$ Department of Mathematics, Faculty of Science, Akdeniz University, Antalya, 07058, Turkey.

\section{Acknowledgements}

Dedicated to Professor Hari M. Srivastava.

All authors are partially supported by Research Project Offices Akdeniz Universities.

Received: 8 November 2012 Accepted: 13 December 2012 Published: 4 January 2013

\section{References}

1. Carlitz, L: Eulerian numbers and polynomials. Math. Mag. 32, 247-260 (1959)

2. Choi, J, Jang, SD, Srivastava, HM: A generalization of the Hurwitz-Lerch zeta function. Integral Transforms Spec. Funct. $19,65-79(2008)$

3. Choi, J, Srivastava, HM: The multiple Hurwitz-Lerch zeta function and the multiple Hurwitz-Euler eta function. Taiwan. J. Math. 15, 501-522 (2011)

4. Choi, J, Kim, DS, Kim, T, Kim, YH: A note on some identities of Frobenius-Euler numbers and polynomials. Int. J. Math. Math. Sci. (2012). doi:10.1155/2012/861797

5. Ding, D, Yang, J: Some identities related to the Apostol-Euler and Apostol-Bernoulli polynomials. Adv. Stud. Contemp. Math. 20, 7-21 (2010)

6. Garg, M, Jain, K, Srivastava, HM: Some relationships between the generalized Apostol-Bernoulli polynomials and Hurwitz-Lerch zeta functions. Integral Transforms Spec. Funct. 17, 803-815 (2006)

7. Gould, HW: The q-series generalization of a formula of Sparre Andersen. Math. Scand. 9, 90-94 (1961)

8. Kim, T: An identity of the symmetry for the Frobenius-Euler polynomials associated with the fermionic $p$-adic invariant $q$-integrals on $\mathbb{Z}_{p}$. Rocky Mt. J. Math. 41, 239-247 (2011)

9. Kim, T: Identities involving Frobenius-Euler polynomials arising from non-linear differential equations. J. Number Theory 132, 2854-2865 (2012). arXiv:1201.5088v1

10. Kim, T, Choi, J: A note on the product of Frobenius-Euler polynomials arising from the $p$-adic integral on $\mathbb{Z}_{p}$. Adv. Stud. Contemp. Math. 22, 215-223 (2012)

11. Kurt, B, Simsek, Y: Frobenious-Euler type polynomials related to Hermite-Bernoulli polynomials. AIP Conf. Proc. 1389, 385-388 (2011)

12. Lin, S-D, Srivastava, HM, Wang, P-Y: Some expansion formulas for a class of generalized Hurwitz-Lerch zeta functions Integral Transforms Spec. Funct. 17, 817-827 (2006)

13. Luo, Q-M: q-analogues of some results for the Apostol-Euler polynomials. Adv. Stud. Contemp. Math. 20, 103-113 (2010)

14. Luo, Q-M, Srivastava, HM: Some generalizations of the Apostol-Genocchi polynomials and the Stirling numbers of the second kind. Appl. Math. Comput. 217, 5702-5728 (2011)

15. Srivastava, HM, Saxena, RK, Pogany, TK, Saxena, R: Integral and computational representation of the extended Hurwitz-Lerch zeta function. Integral Transforms Spec. Funct. 22, 487-506 (2011)

16. Simsek, Y: Generating functions for q-Apostol type Frobenius-Euler numbers and polynomials. Axioms 1, 395-403 (2012). doi:10.3390/axioms1030395

17. Simsek, Y: Generating functions for generalized Stirling type numbers, array type polynomials, Eulerian type polynomials and their application. arXiv:1111.3848v2 
18. Simsek, Y, Kim, T, Park, DW, Ro, YS, Jang, LC, Rim, SH: An explicit formula for the multiple Frobenius-Euler numbers and polynomials. JP J. Algebra Number Theory Appl. 4, 519-529 (2004)

19. Srivastava, HM: Some generalizations and basic (or $q^{-}$) extensions of the Bernoulli, Euler and Genocchi polynomials. Appl. Math. Inform. Sci. 5, 390-444 (2011)

20. Srivastava, HM, Kim, T, Simsek, Y: q-Bernoulli numbers and polynomials associated with multiple q-zeta functions and basic L-series. Russ. J. Math. Phys. 12, 241-268 (2005)

21. Srivastava, HM, Choi, J: Series Associated with the Zeta and Related Functions. Kluwer Academic, Dordrecht (2001)

22. Srivastava, HM, Garg, M, Choudhary, S: A new generalization of the Bernoulli and related polynomials. Russ. J. Math. Phys. 17, 251-261 (2010)

23. Srivastava, HM, Garg, M, Choudhary, S: Some new families of the generalized Euler and Genocchi polynomials. Taiwan. J. Math. 15, 283-305 (2011)

24. Srivastava, HM, Choi, J: Zeta and q-Zeta Functions and Associated Series and Integrals. Elsevier, Amsterdam (2012)

doi:10.1186/1687-1847-2013-1

Cite this article as: Kurt and Simsek: On the generalized Apostol-type Frobenius-Euler polynomials. Advances in Difference Equations 2013 2013:1

\section{Submit your manuscript to a SpringerOpen ${ }^{\ominus}$ journal and benefit from:}

- Convenient online submission

Rigorous peer review

- Immediate publication on acceptance

- Open access: articles freely available online

- High visibility within the field

- Retaining the copyright to your article 